\title{
The ILC as a natural SUSY discovery machine and precision microscope: From light higgsinos to tests of unification
}

\author{
Howard Baer \\ University of Oklahoma, Norman, OK 73019, USA \\ E-mail: baereou.edu
}

Mikael Berggren, Suvi-Leena Lehtinen, Jenny List, Kollassery Swathi Sasikumar*t

Deutsches Elecktronen Synchrotron, Hamburg

E-mail: mikael.berggren@desy.de, suvi-leena.lehtinen@desy.de, jenny.list@desy.de, swathi.sasikumar@desy.de

Keisuke Fujii, Jacqueline Yan

KEK, Tsukuba, Japan

E-mail: keisuke.fujii@kek.jp, jackie@post.kek.jp

\section{Tomohiko Tanabe}

International Centre for Elementary Particle Physics, University of Tokyo, Japan.

E-mail: tomohiko@icepp.s.u-tokyo.ac.jp

\begin{abstract}
The requirement of electroweak naturalness in simple supersymmetric models motivates the existence of a cluster of four light higgsinos with mass $100-300 \mathrm{GeV}$, the lighter the better. While such light compressed spectra may be challenging to observe at the LHC, future $e^{+} e^{-}$colliders with $\sqrt{s}>2 \mathrm{~m}$ (higgsino) would serve as both a SUSY discovery machine and a precision microscope. We study signatures of higgsino pair production at the ILC based on full, Geant4-based simulation of the ILD detector concept. We examine several benchmark scenarios that may or may not be accessible to HL-LHC searches, with mass differences between the higgsino states between 4 and $20 \mathrm{GeV}$. Assuming $\sqrt{s} \geq 500 \mathrm{GeV}$ and $1000 \mathrm{fb}^{-1}$ of integrated luminosity, the individual higgsino masses can be measured to $1-2 \%$ precision in case of the larger mass differences, and at the level of $5 \%$ for the smallest mass difference case. The higgsino mass splittings are sensitive to the electroweak gaugino masses and can allow extraction of gaugino masses to $3-20 \%$ (depending on the model). Extrapolation of gaugino masses via renormalization group running can test the hypothesis of gaugino mass unification. We also examine a case with natural generalized mirage mediation where the unification of gaugino masses at an intermediate scale apparently gives rise to a natural SUSY spectrum somewhat beyond the reach of the HL-LHC.
\end{abstract}

European Physical Society Conference on High Energy Physics - EPS-HEP2019 -

10-17 July, 2019

Ghent, Belgium

\footnotetext{
*Speaker.

${ }^{\dagger}$ On behalf of ILD concept group.
} 


\section{Motivation}

The Standard-Model-like Higgs boson was discovered by the LHC in 2012 [1]. However, due to the unstable quantum corrections to its mass it is quite difficult to comprehend its existence in the Standard Model. Supersymmetry (SUSY) by introducing super-partners for each standard model particle, successfully cancels the quadratic divergent terms thus predicting the mass of Higgs to be $m_{H} \sim 115-135 \mathrm{GeV}$. However, the absence of SUSY discovery in high energy experimental facilities has developed large degree of skeptisism among the physicists thus rekindling the problem of fine-tuning. The connection between the magnitude of the weak scale and SUSY Lagrangian parameters arises from the scalar potential minimization condition [3]:

$$
\frac{m_{Z}^{2}}{2}=\frac{\left(m_{H_{d}}^{2}+\Sigma_{d}^{d}\right)-\left(m_{H_{u}}^{2}+\Sigma_{u}^{u}\right) \tan ^{2} \beta}{\tan ^{2} \beta-1}-\mu^{2} \approx-m_{H_{u}}^{2}-\Sigma_{u}^{u}-\mu^{2}
$$

The issue of fine-tuning can be avoided if the $\mu$ parameter is of the $\mathscr{O}(100-300) \mathrm{GeV}$ (around $m_{\text {weak }} \sim 100 \mathrm{GeV}$ ). As $\mu$ governs the physical mass of higgsinos, it is required that their masses are around $100-300 \mathrm{GeV}$ whereas the rest of the spectra may lie in the multi-TeV range [2].

The radiatively-driven natural SUSY models have light higgsinos which are almost mass degenerate $(\Delta M<20 \mathrm{GeV})$. Probing such processes is quite challenging for hadron colliders like the LHC. With the clean and precise measurements that a lepton collider can provide, there is a prospect to probe such light higgsinos.

The International Linear Collider (ILC) is a proposed $e^{+} e^{-}$collider planned to operate at a $\sqrt{s}=250 \mathrm{GeV}$ and upgradable to $\sqrt{s}=500-1000 \mathrm{GeV}$. The experiment would run with polarized electron and positron beams. The study presented in this proceedings estimates the potential of the ILC for the measurement of higgsinos. It also tests the capacity of the experiment to predict the unobserved sparticles at high energy scales.

\section{Comparison of higgsino measurements with different Natural SUSY models}

Five different benchmark scenarios given as ILC1, ILC2, nGMM1, dM770 and dM1600 are studied and presented in this proceeding. An $e^{+} e^{-}$collider might be able to produce higgsinos like $\tilde{\chi}_{1}^{0}, \tilde{\chi}_{2}^{0}$ and $\tilde{\chi}_{1}^{ \pm}$at a centre-of-mass energy of $500 \mathrm{GeV}$. The mass differences between $\tilde{\chi}_{1}^{ \pm}$and the LSP and $\tilde{\chi}_{2}^{0}$ and the LSP are given in table 1.

\begin{tabular}{|c|c|c|c|}
\hline Models & Benchmark & $\Delta M_{\tilde{\chi}_{1}^{ \pm}}[\mathrm{GeV}]$ & $\Delta M_{\tilde{\chi}_{1}^{0}}[\mathrm{GeV}]$ \\
\hline \hline NUHM1 & ILC1 & 14.6 & 21.3 \\
\hline NUHM2 & ILC2 & 10.2 & 9.7 \\
\hline Mirage mediation & nGMM1 & 7.3 & 4.4 \\
\hline Mixed & dM770 & 0.77 & 1.04 \\
guage-gravitation & dM1600 & 1.6 & 2.7 \\
\hline
\end{tabular}

Table 1: Different benchmark scenarios and mass differences between $\tilde{\chi}_{1}^{ \pm}$and the LSP and $\tilde{\chi}_{2}^{0}$ and the LSP are given. 
The higgsino events are generated by Whizard 1.95 [4] taking the ILC beam spectrum and ISR into account. The produced events are simulated using the Geant 4 [5] based model of the International Large Detector (ILD), which is one of the proposed detectors for the ILC. The centre-of-mass energy is $500 \mathrm{GeV}$ and beam polarization of $80 \%$ for the electrons and $30 \%$ for the positrons with opposite chirality $( \pm 80 \%, \mp 30 \%)$ is assumed.

\subsection{ILC1, ILC2 and nGMM1}

The processes studied for ILC1, ILC2 and nGMM1 are chargino pair production $\left(e^{+} e^{-} \rightarrow\right.$ $\left.\tilde{\chi}_{1}^{ \pm} \tilde{\chi}_{1}^{\mp} \rightarrow \tilde{\chi}_{1}^{0} q \bar{q} \tilde{\chi}_{1}^{0} e v_{e}\left(\mu v_{\mu}\right)\right)$ and neutralino pair production $\left(e^{+} e^{-} \rightarrow \tilde{\chi}_{1}^{0} \tilde{\chi}_{2}^{0} \rightarrow \tilde{\chi}_{1}^{0} \tilde{\chi}_{1}^{0} e^{+} e^{-}\left(\mu^{+} \mu^{-}\right)\right)$.

Since the energies of the initial state particles are known, the higgsino masses can be directly reconstructed from the end points of the invariant mass distributions of the charginos' and neutrilinos' decay products. The maximum endpoints of the di-jet energy $\left(E_{i j}\right)$ and mass $\left(M_{i j}\right)$ distributions for chargino channel and of the di-lepton energy $\left(E_{i j}\right)$ and mass $\left(M_{i j}\right)$ distributions are extracted using a fit as shown in Figure 1 and Figure 2.

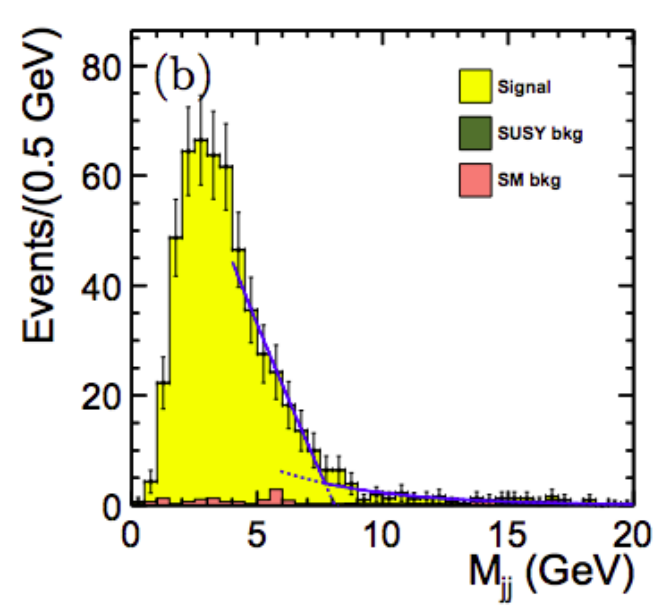

(a)

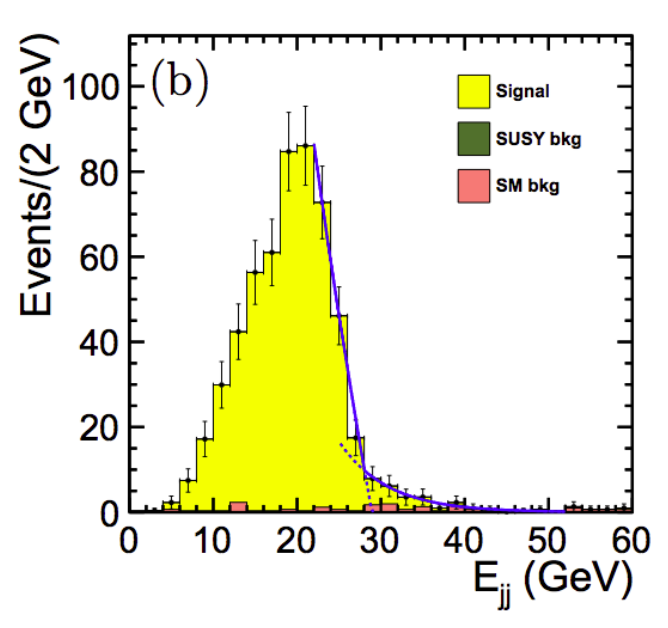

(b)

Figure 1: Endpoint extraction of di-jet invariant mass system's (a)invariant mass and (b) energy distributions in the ILC2 benchmark: neutralino channel, muon final state, beam polarizations $P\left(e^{-}, e^{+}\right)=(-0.8,+0.3)$.

Assuming an integrated luminosity of $500 \mathrm{fb}^{-1}$ for each of the two beam polarizations, the chargino and neutralino masses can be measured with an uncertainity of $0.5-0.7 \%$ for benchmarks with mass gaps of $10 \mathrm{GeV}$ and larger and better than $1 \%$ for benchmarks with mass gaps of few $\mathrm{GeV}$. The cross-section times branching ratio can be measured with a precision of $2-5 \%$ depending on the process, polarization and benchmark model.

\section{$2.2 \mathrm{dM770}$ and $\mathrm{dM1600}$}

In the benchmark models dM770 and dM1600, chargino pair production $\left(e^{+} e^{-} \rightarrow \tilde{\chi}_{1}^{ \pm} \tilde{\chi}_{1}^{\mp} \gamma \rightarrow\right.$ $\left.\tilde{\chi}_{1}^{0} \pi \tilde{\chi}_{1}^{0} e v_{e}\left(\mu v_{\mu}\right) \gamma\right)$ and neutralino pair production $\left(e^{+} e^{-} \rightarrow \tilde{\chi}_{1}^{0} \tilde{\chi}_{2}^{0} \gamma \rightarrow \tilde{\chi}_{1}^{0} \tilde{\chi}_{1}^{0} e^{+} e^{-}\left(\mu^{+} \mu^{-}\right) \gamma\right)$ are 


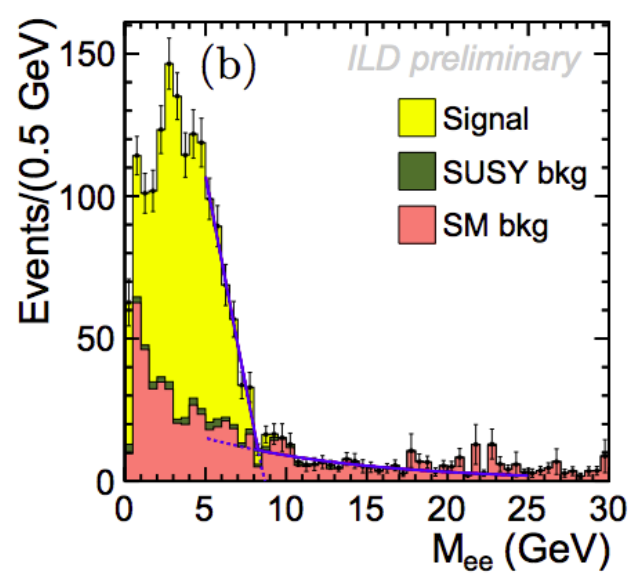

(a)

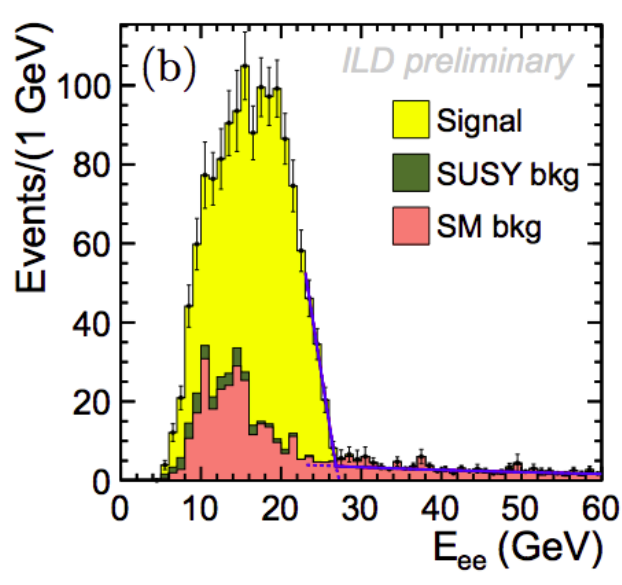

(b)

Figure 2: Endpoint extraction of di-lepton invariant mass system's (a)invariant mass and (b) energy distributions in the ILC2 benchmark: neutralino channel, electron final state, beam polarizations $P\left(e^{-}, e^{+}\right)=(-0.8,+0.3)$.

studied [6]. Unlike for ILC1, ILC2 and nGMM1 benchmark models, chargino process here produces a pion instead of a di-jet. Due to the presence of a hard intial state radiation (ISR) photon, the mass of the higgsinos is not reconstructed using the invariant mass of decay products. Instead, the recoil mass of the system against the hard ISR photon is determined and higgsino mass is extracted by applying a linear fit to the distribution near the endpoint (Figure 3).

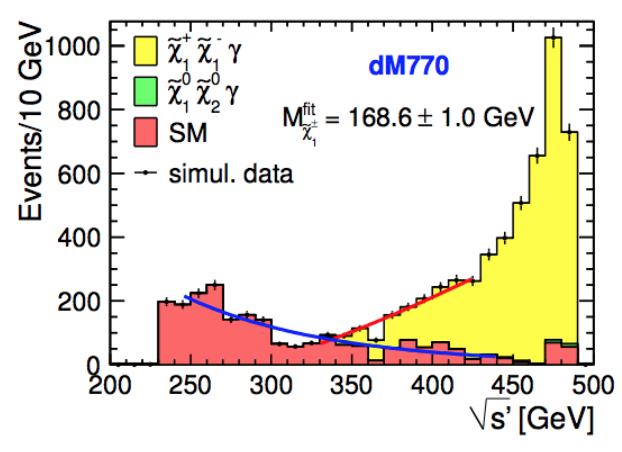

(a)

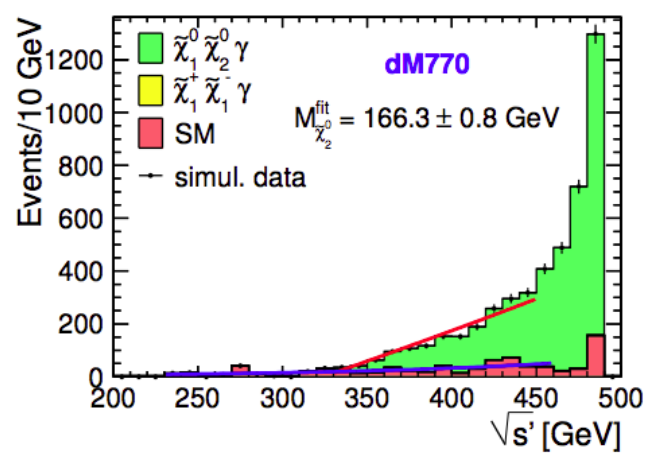

(b)

Figure 3: Endpoint extraction of reduced centre-of-mass energy $\left(\sqrt{s^{\prime}}\right)$ of system recoil against the hard ISR photon $\int \mathscr{L} d t=500 \mathrm{fb}^{-1}$ and beam polarizations $P\left(e^{-}, e^{+}\right)=(-0.8,+0.3)$. (a) $\mathrm{dM} 770$ for Chargino. (b) dM770 for Neutralino. Figure taken from [12].

The neutralino mass can be obtained with a precision of $0.004-0.009 \%$ and the chargino mass can be obtained with a precision of $0.005-0.008 \%$ for an integrated luminosity of $\int \mathscr{L} d t=500$ $\mathrm{fb}^{-1}$ and beam polarizations $P\left(e^{-}, e^{+}\right)=(-0.8,+0.3)$. The cross section times branching ratio is evaluated for neutralinos with a precision of $1.7-3.7 \%$ and for charginos with a precision of $1.6-5.3 \%$ depending on the polarization and the benchmark scenario. 


\section{SUSY parameter fits to measurements at weak scale}

The obtained higgsino measurements can be used to fit SUSY model parameters to get an understanding about the yet unobeserved, higher mass sparticles. This is done by using the fitting algorithm Fittino [7] which uses Markov chain Monte-Carlo to probe the SUSY parameter space [8]. The higgsino masses and cross section measurements along with the Higgs mass and coupling measurements [9] are used as fit inputs. The SUSY spectrum is calculated using Spheno 3.3. 9 beta [10] and the Higgs observables are obtained using FeynHiggs 2.10.2 [11]. A 10 -parameter pMSSM model is fitted using $M 1, M 2, M 3, \tan \beta, \mu, m_{A}, M_{Q}(3), M_{U}(3)$, a common sfermion mass $=M_{Q}(1,2)=M_{U}(1,2)=M_{D}(1,2,3)=M_{L}(1,2,3)$ and a common trilinear coupling $A_{t}=A_{b}=A_{\tau}$ as free parameters. The first and second generation flavour mixing and trilinear coupling is set to zero. The set of ten parameters is fitted and constraints on them are obtained. Constraints on parameters like $M 1, M 2, \mu$ and $\tan \beta$ alone can be obtained by fixing the other six parameters to the best fit values. The fitted parameters can be seen in Figure 5.

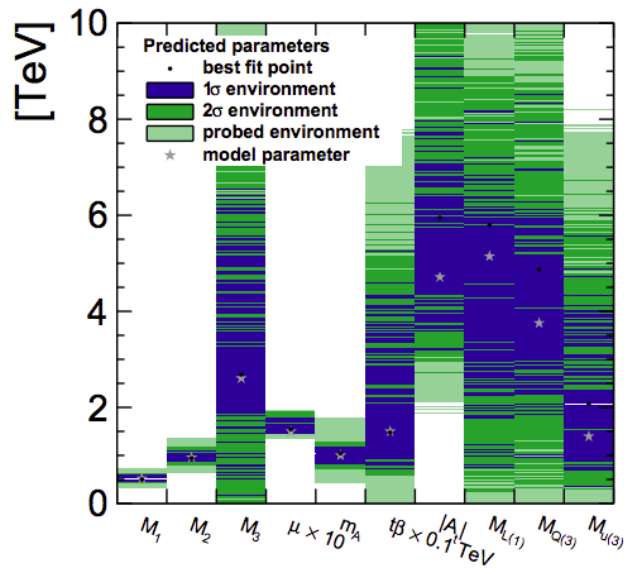

(a)

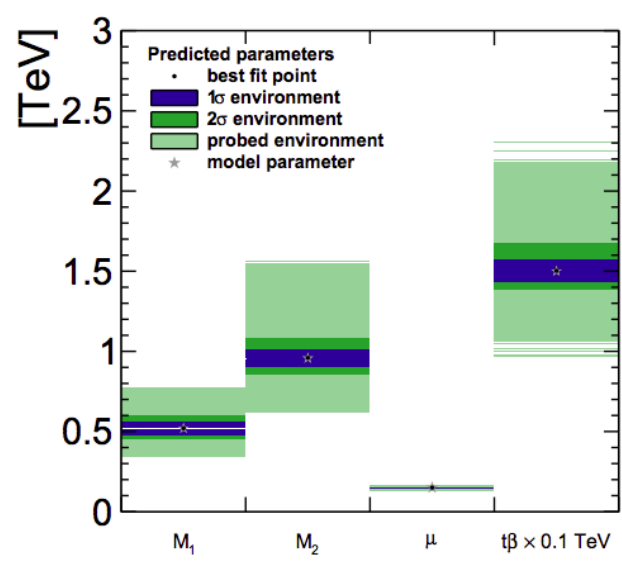

(b)

Figure 4: (a) A set of 10 parameters fitted to SUSY model. (b) 4 parameters fitted by fixing the other six parametrs to best fit values. The grey star indicates the true model values while the black dot shows the best fit point.

As a result the bino and wino mass parameters can be predicted with a precision of $M 1$ up to $3-10 \%$ and $M 2$ up to $2-12 \%$. The higgsino mass parameter $\mu$ can be determined to a precision of $0.3 \%$ and $\tan \beta$ to $1-4 \%$.

Heavy chargino $\left(\tilde{\chi}_{2}^{ \pm}\right)$and neutralino $\left(\tilde{\chi}_{3}^{0}, \tilde{\chi}_{4}^{0}\right)$ masses can be predicted with $m_{\tilde{\chi}_{3}^{0}}$ up to $5-12 \%$ precision and $\tilde{\chi}_{2}^{ \pm}=\tilde{\chi}_{4}^{0}$ up to $4-14 \%$. The predicted mass ranges for all the 10 parameters and gauginos can be seen in Figure 6. The masses vary from $250 \mathrm{GeV}$ to $1800 \mathrm{GeV}$ which forms a motivation for energy upgrade of the ILC or for the building of high energy facilities in the future.

\section{Probing the GUT scale Unification}

SUSY parameters change with scale governed by a system of coupled differential equations called renormalization group equations (RGE). The fitted values are given as an input to RGE's 


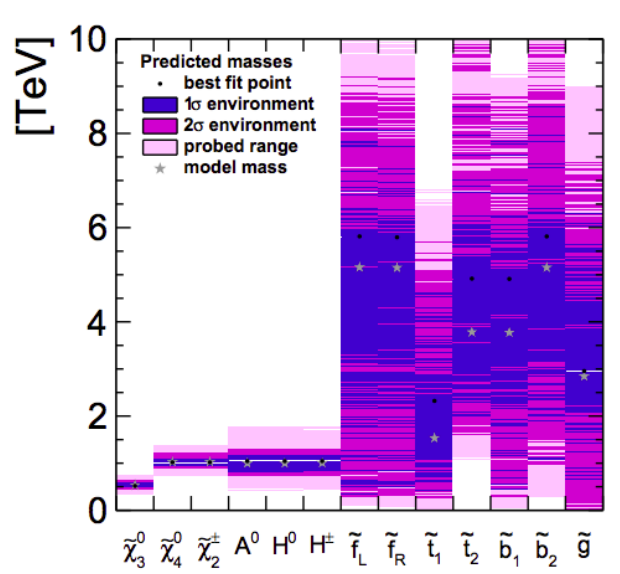

(a)

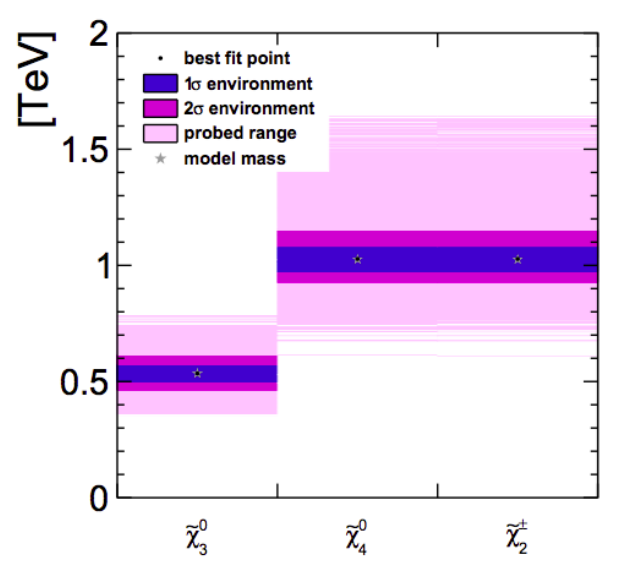

(b)

Figure 5: (a) Predicted mass ranges from the pMSSM-10 fit to ILC2. (b) Fitted Guagino masses. The grey star indicates the true model values while the black dot shows the best fit point.

through Spheno and the three gaugino parameters $M 1, M 2$ and $M 3$ are run to the GUT scale. The gaugino masses unify at the GUT scale as can be seen in Figure 7a. Here, M3 as constrained from the ILC measurements at the weak scale is taken as an input. Instead if it is assumed that $M 3$ unifies with $M 1$ and $M 2$ at the GUT scale, then the value of $M 3$ at the weak scale can be extrapolated to $M 3(Q=1 \mathrm{TeV})=2616 \pm 582 \mathrm{GeV}$ while the physical gluino mass is found to be $m_{\tilde{g}}=2872 \pm 605$ $\mathrm{GeV}$ (Figure 7b).

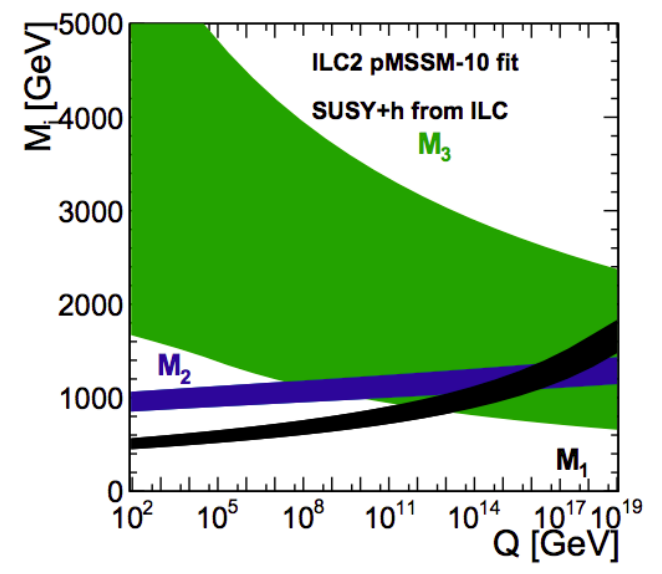

(a)

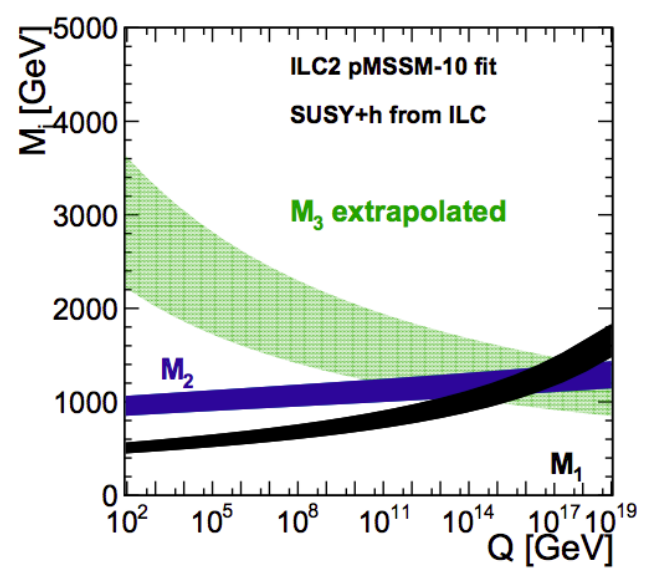

(b)

Figure 6: The running of gaugino masses after extracting their weak scale values from a fit to ILC2 observables. The bands correspond to one standard deviation. (a) Using M3 at the weak scale as contrained from ILC measurements. (b) M3 is assumed to unify with M1 and M2 and M3 is ran back to the weak scale to predict the gluino mass.

With the GUT-scale probed, a further investigation for differentiating the different benchmark models is done. ILC1 and ILC2 benchmark scenarios are compared with nGMM1 benchmark 
model. The running of gaugino mass parameters for ILC1 and ILC2 parameters differ from the running of nGMM1 parameters. This is mainly because nGMM1 is a mirage unification model where the gaugino mass parameters unify at an intermediate energy scale. And secondly, the measurement of $M 1$ and $M 2$ from the weak scale fits is much less accurate in nGMM1 as compared to ILC1 and ILC2 benchmark models. This can be seen in Figure 8.

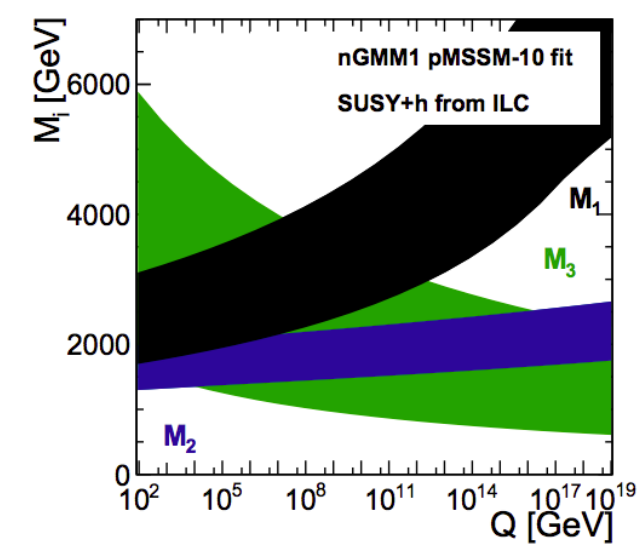

(a)

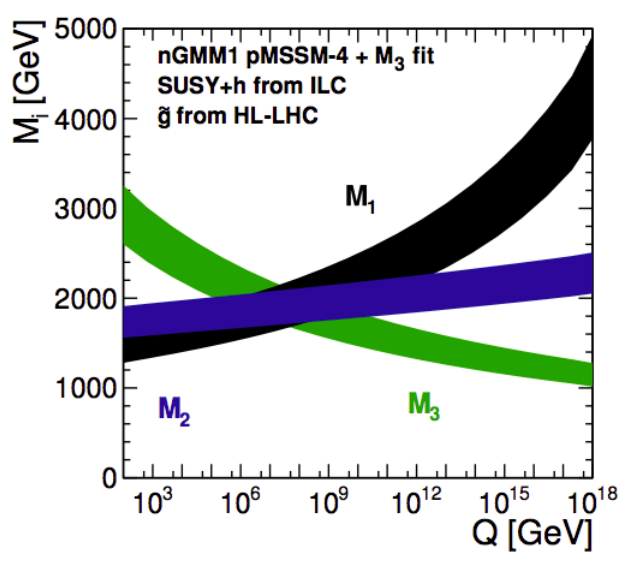

(b)

Figure 7: The running guagino masses after extracting their weak scale values from a fit to nGMM1 observables. The bands correspond to one standard deviation. (a) pMSSM-10 fit result with absolute masses as result (b) estimated effect of improvement with the use of full simulation results.

\section{Conclusion}

Light higgsinos from SUSY can be expected if naturalness arguments about the $\mu$ parameter hold. It is shown that the ILC as a complementary machine to the LHC could provide excellent prospects for both SUSY discovery and precision measurements of sparticles. The ILC could measure the properties of higgsinos to percent-level precision. With the precise measurements, GUT and weak scale parameters can be extracted and mass scales of unobserved sparticles can be predicted.

\section{Acknowledgements}

We would like to thank the LCC generator working group and the ILD software working group for providing the simulation and reconstruction tools and producing the Monte Carlo samples used in this study. This work has benefited from computing services provided by the ILC Virtual Organization, supported by the national resource providers of the EGI Federation and the Open Science GRID. 


\section{References}

[1] G. Aad et al. [ATLAS Collaboration], "Observation of a new particle in the search for the Standard Model Higgs boson with the ATLAS detector at the LHC”, Phys. Lett. B 716 (2012) 1 doi:10.1016/j.physletb.2012.08.020 [arXiv:1207.7214 [hep-ex]].

[2] H. Baer, V. Barger, P. Huang, D. Mickelson, A. Mustafayev and X. Tata, "Radiative natural supersymmetry: Reconciling electroweak fine-tuning and the Higgs boson mass," Phys. Rev. D 87 (2013) no.11, 115028 doi:10.1103/PhysRevD.87.115028 [arXiv:1212.2655 [hep-ph]].

[3] S. P. Martin, “A Supersymmetry primer,” Adv. Ser. Direct. High Energy Phys. 21 (2010) 1 [hep-ph/9709356].

[4] W. Kilian, “WHIZARD manual,” LC-TOOL-2001-039.

[5] S. Agostinelli et al. [GEANT4 Collaboration], "GEANT4: A Simulation toolkit," Nucl. Instrum. Meth. A 506 (2003) 250. doi:10.1016/S0168-9002(03)01368-8

[6] M. Berggren, F. Brümmer, J. List, G. Moortgat-Pick, T. Robens, K. Rolbiecki and H. Sert, “Tackling light higgsinos at the ILC," Eur. Phys. J. C 73 (2013) no.12, 2660 doi:10.1140/epjc/s10052-013-2660-y [arXiv:1307.3566 [hep-ph]].

[7] P. Bechtle, K. Desch and P. Wienemann, "Fittino, a program for determining MSSM parameters from collider observables using an iterative method," Comput. Phys. Commun. 174 (2006) 47 doi:10.1016/j.cpc.2005.09.002 [hep-ph/0412012].

[8] S. L. Lehtinen et al. [ILD concept group], "Naturalness and light Higgsinos: why ILC is the right machine for SUSY discovery,” PoS EPS -HEP2017 (2017) 306 doi:10.22323/1.314.0306 [arXiv:1710.02406 [hep-ph]].

[9] K. Fujii et al., "Physics Case for the International Linear Collider," arXiv:1506.05992 [hep-ex].

[10] W.Porod Comput. Phys. Commun. 153, 275 (2003) [arXiv:hep-ph/0301101].

[11] S. Heinemeyer, W. Hollik and G. Weiglein, "FeynHiggs: A Program for the calculation of the masses of the neutral CP even Higgs bosons in the MSSM," Comput. Phys. Commun. 124 (2000) 76 doi:10.1016/S0010-4655(99)00364-1 [hep-ph/9812320].

[12] H. Sert, "Light Higgsinos at the ILC: Precision Measurements and Detector Requirements," doi:10.3204/DESY-THESIS-2016-001 